\title{
Confinement from Correlation Functions
}

\author{
Leonard Fister* \\ Department of Mathematical Physics, National University of Ireland Maynooth, Maynooth Co. \\ Kildare, Ireland, and \\ Institut für Theoretische Physik, Universität Heidelberg, Philosophenweg 16, 69120 Heidelberg, \\ Germany. \\ E-mail: leonardethphys.nuim.ie
}

\section{Jan M. Pawlowski}

Institut für Theoretische Physik, Universität Heidelberg, Philosophenweg 16, 69120 Heidelberg, Germany, and

ExtreMe Matter Institute EMMI, GSI, Planckstr. 1, D-64291 Darmstadt, Germany.

E-mail: j.pawlowski@thphys.uni-heidelberg.de

\begin{abstract}
We study quark confinement by computing the Polyakov loop potential in Yang-Mills theory within different non-perturbative functional continuum approaches. We extend previous studies in the formalism of the functional renormalisation group and complement those with findings from Dyson-Schwinger equations and two-particle-irreducible functionals. These methods are formulated in terms of low order Green functions. This allows to identify a criterion for confinement solely in terms of the low-momentum behaviour of correlators.
\end{abstract}

Xth Quark Confinement and the Hadron Spectrum,

October 8-12, 2012

TUM Campus Garching, Munich, Germany

${ }^{*}$ Speaker. 


\section{Introduction}

In recent years much progress has been made in our understanding of the strongly-correlated low energy regime of QCD in terms of gauge-fixed correlation functions. This progress is tightly linked to the advance in our understanding of the basic phenomena of low energy QCD, strong chiral symmetry breaking and confinement.

Confinement denotes the absence of colour charged asymptotic states. For infinitely heavy quarks this can be related to the free energy of a single quark $F_{q}$ : Bringing a single quark into the system would require an infinite amount of energy in the confined phase, whereas a finite energy would suffice in the deconfined phase. The operator which creates a static single quark is related to the Polyakov loop $L$,

$$
L=\frac{1}{N_{\mathrm{c}}} \operatorname{tr}_{\mathrm{f}} \mathscr{P} e^{\mathrm{ig} \int_{0}^{\beta} d x_{0} A_{0}\left(x_{0}, x\right)}, \quad \text { and }\langle L\rangle \sim \exp ^{-F_{q} / T},
$$

with the trace in the fundamental representation of $S U\left(N_{c}\right)$ of the closed Wilson line in time direction. Here, $\mathscr{P}$ denotes path ordering, $A$ is the gauge field and the inverse temperature $\beta=1 / T$. This links the confinement-deconfinement phase transition in the Yang-Mills system to the orderdisorder phase transition of center symmetry $Z_{N}$ in $S U\left(N_{c}\right)$ : Under center transformations $z \in Z_{N}$ the Polyakov loop transforms with $L \rightarrow z L$ in the fundamental representation. In the centersymmetric, confining phase the order parameter vanishes, $\langle L\rangle=0$, while in the center-broken, deconfined phase it is finite, $\langle L\rangle \neq 0$.

It has been shown that $L\left[\left\langle A_{0}\right\rangle\right]$ also constitutes an order parameter for static quark confinement $[1,2]$, as $L\left[\left\langle A_{0}\right\rangle\right] \geq\left\langle L\left[A_{0}\right]\right\rangle$ in the deconfined but strictly $L\left[\left\langle A_{0}\right\rangle\right]=0$ in the confined phase. However, $L\left[\left\langle A_{0}\right\rangle\right]$ is easily accessible within functional continuum methods, as $\left\langle A_{0}\right\rangle$ can be straightforwardly computed from the equation of motion, that is via the full effective potential $V\left[A_{0}\right]$. It was shown within the functional renormalisation group (FRG) approach $[1,2,3]$, that $V\left[A_{0}\right]$ has a closed representation in terms of the full propagators of the gluons, ghosts (and quarks) in constant $A_{0}$-backgrounds. With this connection a confinement criterion in terms of the infrared behaviour of the propagators was derived [1].

By now $\left\langle L\left[A_{0}\right]\right\rangle$ and the effective Polyakov loop potential $V\left[A_{0}\right]$ has been computed in YangMills theory in the Landau gauge [1,4] and in the Polyakov gauge [2]. The potential obtained in different gauges agree well which provides a check of the gauge independence. Recently, these studies have been extended to the Coulomb gauge [5]. By now, also lattice results for the potential are available [6, 7]. In [3] the Yang-Mills studies have been extended to fully dynamical twoflavour QCD. In $[8,9], V\left[A_{0}\right]$ is used in an effective model approach to QCD with interesting applications to thermodynamic observables.

Here report on work, in which we compute the Polyakov loop effective potential $V\left[A_{0}\right]$ in the background field formalism in Landau-deWitt gauge within different functional approaches, [10]. In Section 2 we derive representations for $V\left[A_{0}\right]$ with the FRG, Dyson-Schwinger equations (DSEs) and two-particle irreducible (2PI) functionals. In Section 3 we deduce a confinement criterion in terms of the propagators: Infrared suppression of gluons but non-suppression of ghosts confines static quarks at sufficiently low temperatures. In Section 4 numerical results for $V\left[A_{0}\right]$ are presented, which are based on the finite temperature propagators computed within the FRG in 


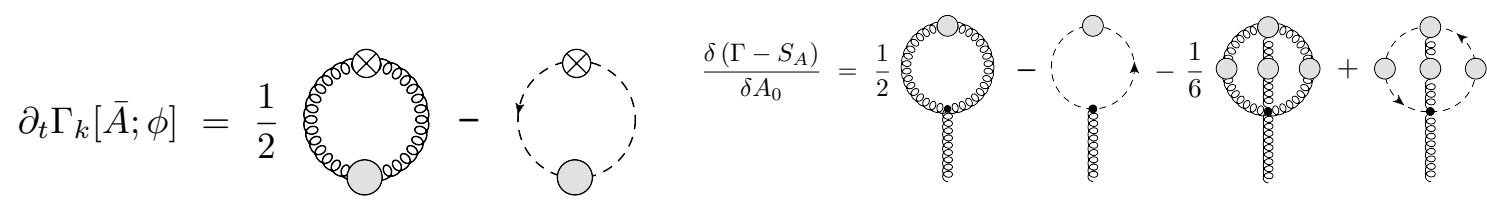

(a) Flow equation for the Polyakov loop potential.

(b) DSE for the background gluon one-point function.

Figure 1: Functional equations for the (derivative of) the Polyakov loop potential. The gluons are represented by wiggly, the ghosts by dashed lines. Filled circles indicate full correlators. Crossed circles denote the regulator insertion $\partial_{t} R_{k}$.

$[11,12,13]$.

\section{Polyakov Loop Potential from Functional Methods}

Most functional approaches are based on closed expressions for the effective action or derivatives thereof in terms of full correlation functions. Hence, the knowledge of the latter in constant $A_{0}$-backgrounds allows us to compute the Polyakov loop potential $V\left[A_{0}\right]$. In turn, confinement requires $V\left[A_{0}\right]$ to have minima at the confining values for $A_{0}$, i.e. in $S U\left(N_{\mathrm{c}}\right)$ the center-symmetric points. With $(\bar{c}) c$ being the (anti-)ghosts, in the background formalism the Polyakov loop potential is the gauge-invariant effective action, $\Gamma\left[A_{0} ; \phi\right]$ with $\phi=\left(a=A-A_{0}, c, \bar{c}\right)$, evaluted on a constant $A_{0}$-background and vanishing fluctuation fields, $V\left[A_{0}^{\text {const }}\right]:=\Gamma\left[A_{0}^{\text {const }} ; 0\right] /(\beta \mathscr{V}) . \mathscr{V}$ is the three-dimensional spatial volume. The Polyakov loop, (1.1), is then computed at the minimum $\left\langle A_{0}\right\rangle:=A_{0, \min }$. Functional equations for the effective action depend on the correlation functions of fluctuation fields only. By gauge covariance the correlation functions in the background Landau gauge, $\Gamma^{(n)}[\bar{A}]$, are directly related to those in the Landau gauge, $\Gamma^{(n)}[0]$. Neglecting subleading corrections, for more details see [10], the two-point functions of gluon and the ghost on constant backgrounds are schematically given by

$$
\Gamma_{a}^{(2)}[A ; 0]=\sum_{L / T}\left(-D^{2}\right) Z_{L / T} P^{L / T}, \text { and } \quad \Gamma_{c}^{(2)}[A ; 0]=\left(-D^{2}\right) Z_{c},
$$

where the $P^{L / T}$ are the projection operators for the chromoelectric/chromomagnetic modes, and $Z_{L / T / c}\left(-D_{0}^{2},-\vec{D}^{2}\right)$ are the wave-function renormalisations of the corresponding gluon and ghost modes evaluated on covariant momenta $D$. The FRG representation of the Polyakov loop potential has been used in $[1,2,4]$. The exact flow equation for the effective action, $\Gamma_{k}[\bar{A} ; \phi]$, is given solely in terms of the propagators,

$$
\partial_{t} \Gamma_{k}[\bar{A} ; \phi]=\sum_{p}\left(\frac{1}{2}\left(G_{a}\right)_{\mu v}^{a b}[\bar{A} ; \phi](p, p) \partial_{t}\left(R_{a}\right)_{v \mu}^{b a}(p)-\left(G_{c}\right)^{a b}[\bar{A} ; \phi](p, p) \partial_{t}\left(R_{c}\right)^{b a}(p)\right)
$$

where the integration and Matsubara summation involve the respective modes. Further, $t=\ln k$, 
with $k$ being a infrared cut-off scale controlled by the regulator $R_{a / c}$. At $k$ the propagators are

$$
G_{a / c, k}(p)=\left(\left(\Gamma_{a / c}\right)_{k}(p)+R_{k}(p)\right)^{-1} .
$$

The diagrammatic representation of the flow equation is given in Fig. 1(a).

Finally, by integration we get the Polyakov loop potential

$$
V\left[A_{0}\right]=V_{k=\Lambda}\left[A_{0}\right]+\frac{1}{\beta \mathscr{V}} \int_{\Lambda}^{0} d t \partial_{t} \Gamma_{k}\left[A_{0} ; 0\right],
$$

where $V_{k=\Lambda}^{\prime}\left[A_{0}\right] \rightarrow 0$ for $\Lambda / T \gg 1$. The DSE for Yang-Mills theory relevant for the Polyakov loop potential is that originating in a derivative of the effective action $\Gamma$ w.r.t. the $A_{0}$-background at fixed fluctuation. It can be written in terms of full propagators and vertices and the renormalised classical action,

$$
\frac{\delta\left(\Gamma\left[A_{0} ; 0\right]-S_{A}\left[A_{0} ; 0\right]\right)}{\delta A_{0}(x)}=\frac{1}{2} S_{A_{0} a a}^{(3)} G_{a}-S_{A_{0} c \bar{c}}^{(3)} G_{c}-\frac{1}{6} S_{A_{0} a a a}^{(4)} G_{a}^{3} \Gamma_{a a a}^{(3)}+S_{A_{0} a c \bar{c}}^{(4)} G_{c}^{2} G_{a} \Gamma_{a c \bar{c}}^{(3)},
$$

with $S_{\phi_{1} \ldots \phi_{n}}^{(n)}$ and $\Gamma_{\phi_{1} \ldots \phi_{n}}^{(n)}$ denoting derivatives of the renormalised classical action $S_{A}$ and effective action with respect to the fields $\phi_{i}$. For constant backgrounds the DSE equation for Polyakov loop potential is given by

$$
\frac{\partial V\left[A_{0}\right]}{\partial A_{0}}=\frac{1}{\beta \mathscr{V}} \frac{\partial \Gamma\left[A_{0} ; 0\right]}{\partial A_{0}},
$$

with (2.5) for the right hand side of (2.6). In comparison with the DSE for fluctuating fields only the vertices differ. This also leads to the emergence of the two-loop diagram involving ghosts. Diagrammatically the DSE is given in Fig. 1(b).

For details on the different representations see [10], where the derivation of the 2PI representation of the Polyakov loop potential shows, that for the purpose presented here it is identical to the DSE form.

\section{Confinement Criterion}

Here we discuss the restriction of the infrared behaviour of the propagators that follows from the FRG. Here, all loops in the full flow, (2.2), effectively boil down to expressions of the form

$$
\sim 4 \pi T \sum_{n \in \mathbb{Z}} \int_{0}^{\infty} \frac{d|\vec{p}|}{(2 \pi)^{3}} \vec{p}^{2} \frac{\partial_{t} R_{k}(x)}{x Z\left(-D_{0}^{2}, \vec{p}^{2}\right)+\Delta m^{2}\left(D, A_{0}\right)+R_{k}(x)},
$$

where $x=-D_{0}^{2}+\vec{p}^{2}$ and $Z=Z_{L}, Z_{T}, Z_{c}$. In (3.1) we do not include the overall minus sign of the ghost contribution. The function $R_{k}$ stands for the scalar parts of the regulators, which are chosen to be $Z$-independent and $O(4)$-symmetric, i.e. $R_{k}(x)=x r\left(x / k^{2}\right)$. The terms $\Delta m^{2}(D, 0)=0$, with $\Delta m^{2}=\Delta m_{L}^{2}, \Delta m_{T}^{2}, \Delta m_{c}^{2}$, are thermal corrections to the Polyakov loop, which disappear with powers of the temperature for $T \rightarrow 0$, and for the present purpose we will neglect these terms, [10],

$$
\Delta m^{2}\left(D, A_{0}\right) \equiv 0 \text {. }
$$


At vanishing temperature we regain $O(4)$-symmetry and $Z\left(-D_{0}^{2}, \vec{p}^{2}\right) \rightarrow Z\left(-D_{0}^{2}+\vec{p}^{2}\right)$. In terms of scaling coefficients $\kappa$ at vanishing temperature,

$$
Z(x)=\mathscr{Z} x^{\kappa}
$$

the corresponding propagators exhibit non-thermal mass gaps $m_{\text {gap }}$ for $\kappa<0$. Computing the expression (3.1) leads to a deconfining potential in $A_{0}$ : Taking a derivative w.r.t. $A_{0}$ it vanishes at $A_{0}=0$ and the center-symmetric points, i.e. where the Polyakov loop vanishes, $L=0$. The second derivative is positive at $A_{0}=0$ while it is negative at the center-symmetric points.

Hence, with (3.2) the gluon loops in the flow equation (2.2) give deconfining potentials for the gluons. In contrast, the ghosts give a confining contribution due to the relative minus sign. Thus, confinement in covariant gauges requires the suppression of gluonic contributions. Indeed, the same mechanism is at work in the DSE representation [10]. With the assumption (3.2) this yields a necessary condition for confinement,

$$
\lim _{x \rightarrow 0} \frac{1}{Z_{L / T}(x)}=0 \text { and } \lim _{x \rightarrow 0} \frac{1}{Z_{c}(x)}>0, \quad \text { thus, } \quad \kappa_{L / T}<0 \text { and } \kappa_{c} \geq 0,
$$

where $\kappa_{L / T}$ are the anomalous dimensions of the chromoelectric and chromomagnetic gluon. In (3.4) the condition involving the ghost guarantees that its contribution dominates the trivial gauge mode. Eq. (3.4) is a sufficient condition for confinement in Yang-Mills propagators (with the assumption (3.2)) as it leads to a vanishing order parameter, the Polyakov loop expectation value. Note also that it has been shown $[14,15]$ within a scaling analysis that $\kappa_{c} \geq 0$ has to hold in the infrared.

The infrared limits (3.4) are satisfied for both decoupling type, i.e. $\kappa_{c}=0$ and $\kappa_{a}=-1$, and scaling type, i.e. $\kappa_{c}=\kappa=-\kappa_{a} / 2$ with $\kappa \approx 0.595$, solutions, for an overview of the different solutions see e.g. [16]. As a consequence, both types exhibit confinement. Furthermore, they do not affect the critical physics at the phase transition, as it is triggered in the mid-momentum regime where both types of solutions agree on a quantitative level.

The confinement criterion provided here leads to a simple counting scheme for general theories, e.g. Yang-Mills-Higgs systems [17, 18, 19, 20, 21], or QCD with fundamental or adjoint quarks, [3, 22, 23, 24].

\section{Results for the Polyakov Loop Potential and $T_{c}$}

Here we present the results for $V\left[A_{0}\right]$ in the FRG, DSE and 2PI approaches, which have previously been computed with the thermal propagators obtained from the FRG formalism [11, 12, 13]. The potential determined from the flow equation (2.2) is depicted for temperatures around the confinement-deconfinement phase transition for $S U(2)$ in Fig. 2(a), and for $S U(3)$ in Fig. 2(b). The critical temperatures $T_{c}$ are determined at

$$
T_{c}^{S U(2)}=230 \pm 23 \mathrm{MeV} \text { and } T_{c}^{S U(3)}=275 \pm 27 \mathrm{MeV}
$$

The absolute temperatures in (4.1) are set in comparison to absolute scales of the lattice propagators $[25,26]$ with a string tension $\sigma=(420 \mathrm{MeV})^{2}$, which leads to the dimensionless ratios of 


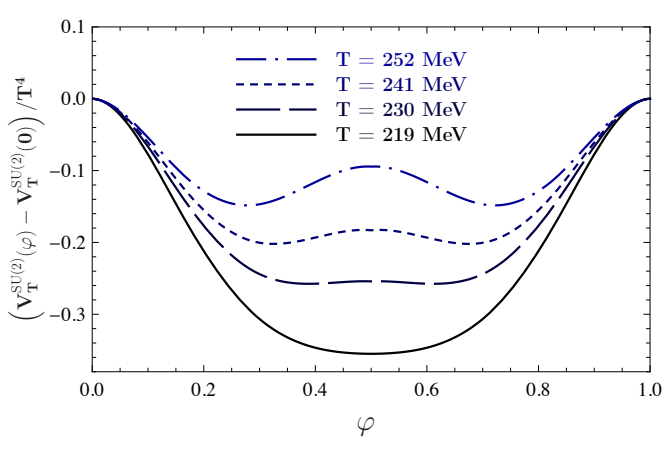

(a) Potential for $S U(2)$.

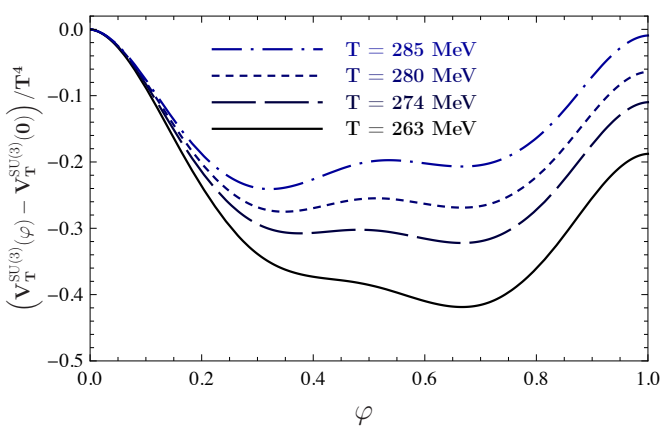

(b) Potential for $S U(3)$.

Figure 2: Polyakov loop potential for $S U(2)$ and $S U(3)$ obtained from the FRG.

$T_{c}^{S U(2)} / \sqrt{\sigma} \approx 0.548$ and $T_{c}^{S U(3)} / \sqrt{\sigma} \approx 0.655$, respectively. For $S U(3)$ our result is in quantitative agreement with the lattice results, see e.g. [27],

$$
T_{c, \text { latt }}^{S U(2)}=295 \mathrm{MeV}, T_{c, \text { latt }}^{S U(3)}=270 \mathrm{MeV}, \text { thus, } \frac{T_{c, \text { latt }}^{S U(2)}}{\sqrt{\sigma}} \approx 0.709, \frac{T_{c, \text { latt }}^{S U(3)}}{\sqrt{\sigma}} \approx 0.646 .
$$

Our relative normalisation is taken from the peak position of the lattice propagators in $[25,26]$. Note however, that this normalisation has an error of approximately $10 \%$, which sets the errors.

In turn, for $S U(2)$ the critical temperature $T_{c}^{S U(2)}$ is significantly lower than the lattice temperature in (4.2). This is linked to the missing back-reaction of the fluctuations of $V\left[A_{0}\right]$ onto the propagators [10]. Its inclusion in the Landau gauge flow leads to a transition temperature of $T_{c}^{S U(2)}=300 \pm 30 \mathrm{MeV}$ [28], in quantitative agreement with results in Polyakov gauge [2] and from lattice gauge theory.

For the computation of $V\left[A_{0}\right]$ from its DSE, (2.6), two-loop diagrams involving dressed vertices in the presence of a background $A_{0}$ need to be determined. However, employing a resummation scheme based on the 2PI-hierachy their contributions can be minimised, which sets the optimal renormalisation point $\mu \approx 1 \mathrm{GeV}$ [10]. As a consequence, it suffices to evaluate a one-loop equation which does not involve dressed Green functions other than the propagators.

The one-loop diagrams in the DSE, Fig. 1(b), reduce to

$$
\begin{aligned}
V^{\prime}(\varphi)= & \frac{1}{2} V_{\text {Weiss }}^{\prime}(\varphi)+\sum_{q} 2 \pi T(n+\varphi) \\
& \times\left[z_{a} G_{L}(q+2 \pi T \varphi)+2 z_{a} G_{T}(q+2 \pi T \varphi)-2 z_{c} G_{c}(q+2 \pi T \varphi)\right],
\end{aligned}
$$

where $G_{L / T}, G_{c}$ are the propagators of the chromoelectric/chromomagnetic gluon and ghost normalised at $z_{a / c}=Z_{a / c}\left(p^{2}=\mu^{2}\right)$ at zero temperature. The first term originates in the trivial gauge mode. The evaluation of (4.3) leads to the potential depicted in Fig. 3(a) for $S U(2)$ and Fig. 3(b) 


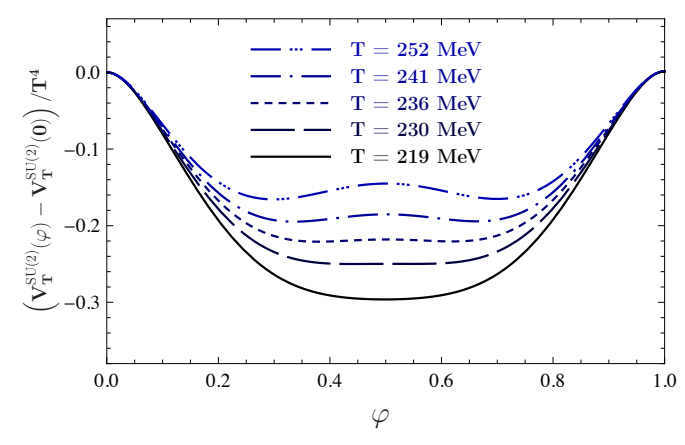

(a) Potential for $S U(2)$.

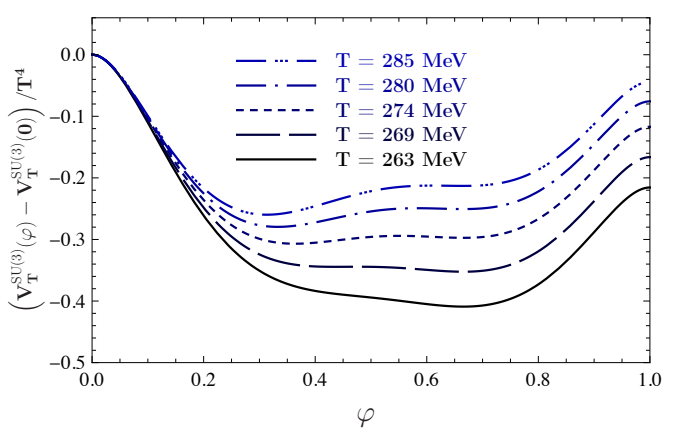

(b) Potential for $S U(3)$.

Figure 3: Polyakov loop potential for $S U(2)$ and $S U(3)$ from its DSE/2PI-representation within an optimised RG-scheme.

for $S U(3)$. From those the confinement-deconfinement critical temperatures are determined at

$$
T_{c}^{S U(2)}=235 \pm 24 \mathrm{MeV}, T_{c}^{S U(3)}=275 \pm 27 \mathrm{MeV}, \text { thus, } \quad \frac{T_{c}^{S U(2)}}{\sqrt{\sigma}} \approx 0.56, \frac{T_{c}^{S U(3)}}{\sqrt{\sigma}} \approx 0.655 .
$$

\section{Conclusions}

We have studied the confinement-deconfinement phase transition of static quarks with the Polyakov loop (effective) potential, which was computed in the background field formalism in the Landau-deWitt gauge [10]. This allowed for the use of Landau gauge propagators at vanishing background, which had been obtained previously in the framework of the FRG [12, 11, 13]. The study here was done within different non-perturbative functional continuum approaches, i.e. using FRG-, DSE- and 2PI-representations of the effective action.

In agreement with lattice gauge theory we find a second and first order phase transition for $S U(2)$ and $S U(3)$, respectively. The critical temperatures in different methods agree quantitatively within errors. These results are stable w.r.t. the choice of scaling or decoupling type solutions for the propagators. The critical temperature for $S U(3)$ from functional methods agrees quantitatively with lattice results. For $S U(2)$ the critical temperature from functional methods is below the lattice temperature, which is due to the missing back-reaction of the fluctuations of the Polyakov loop potential onto the higher Green functions, in particular the chromoelectric propagator.

The dependence of the Polyakov loop potential on the individual gluon and ghost modes was exploited to derive a simple criterion for static quark confinement. For infrared suppressed gluon but not suppressed ghost propagators confinement is immanent at sufficiently low temperatures, as the transversal gluons do not contribute and the ghost modes overcompensate the deconfining longitudinal gluon mode.

In summary this work establishes the functional approach to confinement with means of the Polyakov loop potential. It only requires the background gauge propagators. Hence, it gives easy 
access to structural questions about the confinement mechanism and to explicit computations of the phase structure.

Acknowledgments - This work is supported by the Helmholtz Alliance HA216/EMMI and by ERC- AdG-290623. LF is supported by the Science Foundation Ireland in respect of the Research Project 11-RFP.1-PHY3193 and by the Helmholtz Young Investigator Grant VH-NG-322.

\section{References}

[1] J. Braun, H. Gies, and J. M. Pawlowski, Phys.Lett. B684, 262 (2010), 0708.2413.

[2] F. Marhauser and J. M. Pawlowski, (2008), 0812.1144.

[3] J. Braun, L. M. Haas, F. Marhauser, and J. M. Pawlowski, Phys.Rev.Lett. 106, 022002 (2011), 0908.0008.

[4] J. Braun, A. Eichhorn, H. Gies, and J. M. Pawlowski, Eur.Phys.J. C70, 689 (2010), 1007.2619.

[5] H. Reinhardt and J. Heffner, Phys.Lett. B718, 672 (2012), 1210.1742.

[6] D. Diakonov, C. Gattringer, and H.-P. Schadler, JHEP 1208, 128 (2012), 1205.4768.

[7] J. Greensite, Phys.Rev. D86, 114507 (2012), 1209.5697.

[8] K. Fukushima and K. Kashiwa, (2012), 1206.0685.

[9] K. Kashiwa and Y. Maezawa, (2012), 1212.2184.

[10] L. Fister and J. M. Pawlowski, (2013), 1301.4163.

[11] L. Fister and J. M. Pawlowski, (2011), 1112.5440.

[12] L. Fister and J. M. Pawlowski, (2011), 1112.5429.

[13] L. Fister, PhD thesis, Heidelberg University (2012).

[14] C. S. Fischer and J. M. Pawlowski, Phys. Rev. D75, 025012 (2007), hep-th/0609009.

[15] C. S. Fischer and J. M. Pawlowski, Phys. Rev. D80, 025023 (2009), 0903.2193.

[16] C. S. Fischer, A. Maas, and J. M. Pawlowski, Annals Phys. 324, 2408 (2009), 0810.1987.

[17] L. Fister, (2010), 1002.1649.

[18] L. Fister, R. Alkofer, and K. Schwenzer, Phys.Lett. B688, 237 (2010), 1003.1668.

[19] V. Macher, Diploma thesis, University of Graz (2010).

[20] R. Alkofer, L. Fister, A. Maas, and V. Macher, AIP Conf.Proc. 1343, 179 (2011), 1011.5831.

[21] V. Macher, A. Maas, and R. Alkofer, Int.J.Mod.Phys. A27, 1250098 (2012), 1106.5381.

[22] J. M. Pawlowski, AIP Conf.Proc. 1343, 75 (2011), 1012.5075.

[23] J. Braun and A. Janot, Phys.Rev. D84, 114022 (2011), 1102.4841.

[24] J. Braun and T. K. Herbst, (2012), 1205.0779.

[25] A. Maas, J. M. Pawlowski, L. von Smekal, and D. Spielmann, Phys.Rev. D85, 034037 (2012), 1110.6340 .

[26] A. Maas, Phys. Rep. in press (2013), 1106.3942.

[27] B. Lucini and M. Panero, (2012), 1210.4997.

[28] F. Spallek, Diploma thesis, Heidelberg University (2010). 